A N N A L E S Annales de Bretagne et des Pays de l'Ouest

\title{
Philippe HAUDRÈRE (dir.), Pour une histoire sociale des villes
}

\section{Géraldine Le Pottier}

\section{OpenEdition}

Journals

Édition électronique

URL : https://journals.openedition.org/abpo/382

DOI : 10.4000/abpo.382

ISBN : 978-2-7535-1509-3

ISSN : 2108-6443

Éditeur

Presses universitaires de Rennes

Édition imprimée

Date de publication : 30 mars 2008

Pagination : 226-230

ISBN : 978-2-7535-0653-4

ISSN : 0399-0826

Référence électronique

Géraldine Le Pottier, "Philippe haudrène (dir.), Pour une histoire sociale des villes », Annales de Bretagne et des Pays de l'Ouest [En ligne], 115-1 | 2008, mis en ligne le 30 mars 2008, consulté le 22 juillet 2022. URL : http://journals.openedition.org/abpo/382 ; DOI : https://doi.org/10.4000/abpo.382

Ce document a été généré automatiquement le 22 juillet 2022.

Tous droits réservés 


\title{
Philippe HAUDRÈRE (dir.), Pour une histoire sociale des villes
}

\author{
Géraldine Le Pottier
}

\section{RÉFÉRENCE}

Rennes, PUR, coll. « Histoire », 2006, 445 p.

\section{NOTE DE L'ÉDITEUR}

Cet ouvrage sur le site des Presses universitaires de Rennes : notice, documents et commande en ligne : http://www.pur-editions.fr/detail.php?idOuv=1332

1 Pour une histoire sociale des villes rassemble vingt-six contributions offertes, par ses amis et collègues, à Jacques Maillard, au terme de sa carrière en tant qu'enseignant, chercheur et administrateur à l'université d'Angers. Si, comme le précise Philippe Haudrère dans l'introduction, ce volume d'hommage est consacré pour moitié à l'histoire de la ville d'Angers, objet privilégié des recherches de Jacques Maillard, il permet également les comparaisons grâce à diverses contributions qui déplacent le regard du lecteur sur l'histoire de d'autres cités (en France mais aussi en Hongrie, en Égypte ou au Canada). Ouvrant la réflexion dans le cadre d'une chronologie qui s'étend de l'Antiquité au $\mathrm{xx}^{\mathrm{e}}$ siècle, cette compilation de monographies ponctuelles offre à la fois un riche aperçu des recherches contemporaines en histoire urbaine et une vision, inscrite dans la longue durée, des évolutions qui se sont produites dans les cinq domaines de réflexion qui structurent l'ouvrage: les hommes et les institutions municipales, l'affirmation de l'autorité des "gens du roi ", la domination de la ville sur le plat pays, la culture des élites urbaine et les pratiques religieuses.

Une première partie, consacrée à l'histoire des municipalités en France, met en exergue les rapports entre les hommes et les institutions municipales. Jean-Michel Matz, à la 
suite des travaux de Jacques Maillard, pose la question du lien entre les élites urbaines et l'exercice de charges municipales de la fin $d u \mathrm{Xv}^{\mathrm{e}}$ au début du XvI ${ }^{\mathrm{e}}$ siècle : à partir d'une comparaison d'études prosopographiques des élites municipales et des notables ecclésiastiques à Angers, il met en évidence les stratégies sociales qui permettent à une élite émergente d'hommes de loi liés au pouvoir ecclésiastique d'accéder aux nouvelles fonctions de maires et d'échevins offertes par la récente création des " corps de ville " dans les cités du royaume. Ces autorités municipales deviennent dès le $\mathrm{xv}^{e}$ siècle les interlocutrices privilégiées du pouvoir royal qui en fait des partenaires essentiels notamment pour la défense du territoire; c'est ce que montre Laurent Bourquin en prenant l'exemple du «jeu d'échange " qui s'établit entre le roi et les cités de Champagne pour la participation à l'effort de guerre au milieu du XvI ${ }^{\mathrm{e}}$ siècle. On peut retenir de la réflexion de Guy Saupin sur le mouvement des octrois à Nantes aux XvI ${ }^{\mathrm{e}}$ et $\mathrm{XVII}^{\mathrm{e}}$ siècles le rôle majeur joué par ces maires et échevins dans le maintien des privilèges fiscaux urbains. À travers l'exemple de l'ascension sociale de la famille Aubry, Claude Petitfrère souligne l'honneur que confère la participation aux fonctions municipales aux XVI ${ }^{\mathrm{e}}$ et $\mathrm{XVIII}^{\mathrm{e}}$ siècles. Benoît Garnot apporte quant à lui un éclairage sur une institution municipale: les prisons urbaines; l'usage intéressant qu'il fait des sources judicaires lui permet alors de tirer des conclusions notamment à propos de l'aire d'influence moyenne de la ville de Beaune sur laquelle il focalise son attention. L'analyse de l'image des municipalités, telle qu'elle est véhiculée à travers les almanachs administratifs et historiques, conduit cependant Véronique Sarrazin à confirmer pour le xviII siècle l'hypothèse d'un certain affaiblissement des municipalités sous la tutelle monarchique. Néanmoins, la trajectoire sociale et politique ascendante d'un négociant de province devenu maire d'Angers, décrite par Jacques-Guy Petit, témoigne du prestige et de l'honneur que confère toujours l'exercice de fonctions municipales au XIX ${ }^{\mathrm{e}}$ siècle.

3 La relation entre le pouvoir central, les autorités locales et les habitants de la cité constitue la question centrale de la deuxième partie. Noël-Yves Tonnerre met dans un premier temps en évidence le tournant qui s'est opéré dans la nature du pouvoir princier au $\mathrm{xIII}^{\mathrm{e}}$ siècle en s'appuyant sur l'exemple de Charles d'Anjou: ce dernier, également roi de Sicile, a pu gérer son territoire à distance grâce à l'intermédiaire d'une solide administration, efficace dans la gestion de la ville aussi bien sur le plan politique que sur le plan économique. L'examen réalisé par Dominique Le Page du cas des Angevins entrés à la Chambre des comptes de Bretagne permet de comprendre les stratégies par lesquelles de 1572 aux guerres de la Ligue, le pouvoir royal s'assure le contrôle d'une province bretonne nouvellement rattachée au royaume, tout en satisfaisant les sollicitations de notables angevins, fidèles à la couronne et intéressés par l'achat de charges en Bretagne. La variation du nombre de notaires royaux à Angers du XVI ${ }^{e}$ au XVIII ${ }^{e}$ siècle, décrite par Philippe Haudrère, témoigne d'autre part de la place croissante que prennent ces hommes du roi dans la société angevine ; le répertoire des notaires fourni dans cet article appelle à une poursuite des recherches sur ce sujet. Quelle évaluation peut-on faire de l'exercice de la justice réalisée par les officiers du roi sous le règne de Louis XIV ? C'est la question à laquelle Claude Michaud tente de répondre grâce à la lecture du questionnaire remplit par l'intendant du Berry à la suite d'une enquête lancée par Colbert : il ressort que les officiers de justice, mus par le souci de préserver l'honneur familial, font, pour la moitié d'entre eux, honorablement leur travail. Le roi de France délègue également des sujets à son service hors de France ; 
c'est le cas du consul de France à Naples dont François Brizay étudie la correspondance: la protection et la défense des intérêts français ainsi que la description des habitants du royaume de Naples sont les principales missions que cet homme du roi réalise consciencieusement. Ce sont les mobilités géographiques et sociales dans la France du xvIII ${ }^{\mathrm{e}}$ siècle qui intéressent Philippe Jarnoux : à travers des exemples bien choisis, il met en évidence les trajectoires sociales opposées d'Angevins qui ont pourtant pour même destination la Bretagne; cette réflexion permet alors de cerner les enjeux liés aux fonctions administratives : d'un côté l'élite angevine convoite l'accès à la Chambre des comptes nantaise, de l'autre le petit peuple d'Anjou, voleurs de bétails et vagabonds, redoutent les sévères condamnations des tribunaux au bagne de Brest.

Une troisième partie est consacrée aux formes de dominations de la ville sur le plat pays. L'extension du pouvoir de la ville sur les campagnes est perceptible à Angers par l'emprise progressive de la ville sur une banlieue qui se déploie du XIII ${ }^{\mathrm{e}}$ au XVIII ${ }^{\mathrm{e}}$ siècle et dont François Comte tente de déterminer les limites grâce à l'étude de sources indirectes liées principalement aux affaires judiciaires. L'autorité de la ville sur le plat pays se manifeste également par l'application des prélèvements fiscaux : à la lumière de l'exploitation d'un document inédit relatif à la révolte des bonnets rouges en 1675 dans le pays de Cornouaille, Michel Nassiet confirme le poids des prélèvements dans le mécontentement qui motive les paysans bretons à se révolter. Antoine Follain et Tony Guéry, dans une réflexion commune sur la question atypique du commerce des crottes et des cendres en Anjou, soulignent l'influence et la domination qu'exercent les hommes de la ville sur les paysans des campagnes environnantes pour des questions agricoles et commerciales. L'article de Jean-Pascal Simonin offre quant à lui un dernier exemple de révolte des ruraux contre la domination des dirigeants politiques des villes à travers l'étude des émeutes frumentaires en Indre au XIX ${ }^{e}$ siècle; ces événements montrent en effet l'opposition qui s'est instaurée entre une élite intellectuelle urbaine prônant le principe de la liberté du commerce des grains et des masses populaires inquiètes des pénuries et des hausses de prix.

5 La culture des élites urbaine est le quatrième thème abordé dans cet ouvrage. La lecture proposée par Jean Tricard du Livre du chevalier de la Tour Landry, seigneur angevin du XIV siècle, permet d'apprécier différents aspects de la « culture des nobles » de la fin du Moyen Âge : ce manuel d'éducation, très lu en Europe, révèle en effet l'étendue de la culture livresque de l'auteur ou encore son goût pour l'histoire et les chroniques, mais aussi ses conceptions quant à l'éducation devant être dispensée aux jeunes filles supposée former des épouses pieuses, humbles et fidèles. L'œuvre de Frain de Tremblay, conseiller au présidial d'Angers et membre fondateur de l'Académie de la province, renseigne sur les préoccupations d'une partie de l'élite angevine du $\mathrm{XVII}^{\mathrm{e}}$ siècle: tendance moralisatrice et intransigeance religieuse sont les deux caractéristiques principales qui ressortent de l'étude réalisée par Frédérique Pitou. Au fil de citations extraites de lettres d'enfants pensionnaires adressées à leurs parents, Serge Chassagne dresse un tableau de la société bourgeoise de la fin du XvIII ${ }^{\mathrm{e}}$ siècle : on peut y lire, parfois dans un langage normé, un respect de valeurs telles que l'argent, le travail et les liens familiaux. Jean-Luc Marais nous entretient, pour cette même période, sur le développement des sociétés de loisirs à Angers notamment : ces divers cercles qui regroupent nobles, propriétaires, négociants, magistrats, mais aussi commerçants et manufacturiers perdurent longtemps après la Révolution qui a cependant fait 
apparaître des clivages politiques durables. Enfin, Sylvain Bertoldi propose une présentation complète et illustrée des pratiques culturelles et sociales de Robert Brisset, passionné de photographie; ces pratiques apparaissent comme l'expression d'une forme d'art et de sociabilité contemporaine à Angers.

Les contributions relatives à la pratique religieuse des citadins constituent la cinquième partie de l'ouvrage. Des noms de villes, étroitement liés aux noms des dieux qui y sont vénérés, aux rituels - dont la curieuse pratique du sacrifice humain par le feu explicitée par Jean-Yves Carrez-Maratray -, la religion occupe déjà une place importante dans les civilisations urbaines de l'Égypte pharaonique. Elle n'est pas moins présente dans la Gaule romaine, comme le laisse supposer la stèle édifiée par un dévot en l'honneur de Mars Loucetius, découverte à Angers et à laquelle Michel Molin consacre un article. Marie-Madeleine De Cevins élargit quant à elle le cadre géographique de la réflexion en prenant l'exemple de la Hongrie pour aborder la question du rôle joué par l'Église catholique dans le secours aux pauvres et aux malades des agglomérations urbaines occidentales du $\mathrm{XIII}^{\mathrm{e}}$ au $\mathrm{XVI}^{\mathrm{e}}$ siècle: jusqu'au $\mathrm{XIV}^{\mathrm{e}}$ siècle, dans un contexte de forte adhésion aux croyances religieuses, l'assistance est largement assurée par les fondations religieuses et ordres hospitaliers; faute de moyens, et malgré une sécularisation progressive des infrastructures hospitalières, la charité reste le moyen principal de secours jusqu'au $\mathrm{XVI}^{\mathrm{e}}$ siècle. Plus tard, au XVIII siècle, l'épisode de la prédication de Jacques Bridaine dans la ville de Montpellier rend compte des efforts réalisés par l'Église catholique pour évangéliser les populations urbaines. Robert Sauzet nous renseigne sur les qualités et l'astuce de cet orateur talentueux qui n'hésitait pas à user d'effets théâtraux pour attiser la peur de l'auditoire et provoquer sa conversion. Une réflexion menée par Jean-Claude Robert sur l'histoire de l'urbanisation de la ville de Montréal permet d'envisager de façon pertinente le rapport entre urbanité et vie religieuse : au milieu du XIX ${ }^{e}$ siècle, alors que la population de Montréal ne cesse de croître, l'évêque Ignace Bourget entreprend en effet un projet de morcellement de l'immense paroisse en de multiples paroisses urbaines offrants aux fidèles à la diversité des services et permettant surtout de favoriser une interaction entre le clergé et ses fidèles. La construction à Cholet en 1937 d'une église vouée au Sacré-Cœur témoigne d'une ferveur religieuse qu'Yves Denéchère se propose d'interpréter en évoquant à la fois le poids des croyances traditionnelles, le contexte troublé de l'entre-deux-guerres mais surtout la dimension symbolique et mémorielle liée aux guerres de Vendée.

7 À travers les réflexions qu'il propose sur la question des institutions, des relations entre la population des villes et celle des campagnes, des pratiques culturelles et religieuses, cet ouvrage contribue au renouvellement des connaissances en histoire des populations citadines et invite, dans son titre même, à la poursuite des recherches, sur la ville d'Angers, et plus généralement en histoire urbaine et rurale, l'une n'allant pas sans l'autre. 